PROCEEDINGS OF THE

AMERICAN MATHEMATICAL SOCIETY

Volume 125, Number 2, February 1997, Pages 393-396

S 0002-9939(97)03825-2

\title{
CONGRUENCES ON "CHARACTER" VALUES OF PERMUTATION SUMMANDS
}

\author{
XIANGYONG WANG
}

(Communicated by Ronald M. Solomon)

\begin{abstract}
A class of congruences on "character" values $\Phi_{L}$ of a permutation summand $L$ are exhibited, from which follows the connectedness of the prime ideal spectrum of the Grothendieck ring of permutation summands.
\end{abstract}

Let $G$ be a finite group and $A$ the ring of integers in a number field $K$. An $A G$-lattice is called a permutation lattice if it has an $A$-basis, necessarily finite, which is permuted by the action of $G$. It will be called a permutation summand (for $G$ over $A$ ), if it is a direct summand, as $A G$-module, of a permutation lattice. The Grothendieck ring $\Omega_{A}(G)$ of the category of all permutation summands for $G$ over $A$ has been studied in [3], via a sort of numerical character $\Phi_{L}$ of a permutation summand $L$. The construction of $\Phi_{L}$ is reviewed in the first paragraph of the proof below.

In this note we exhibit a class of congruences on the values of $\Phi_{L}$ which are strong enough to imply the connectedness of the prime ideal spectrum of $\Omega_{A}(G)$. The corresponding result for the character ring $R_{K}(G)$ was established in [2], where Lemma 7 gives analogous congruences on character values. For the Burnside ring $\Omega(G)$ of finite $G$-sets, the connectedness fails [1], because there are too few congruences on the number of fixed points of $G$-sets.

The function $\Phi_{L}$ takes values in the ring $A^{\prime}$ of integers of some sufficiently large number field, for instance $K\left(\zeta_{|G|}\right)$, and is defined on triples $\left(H, b, \mathfrak{p}^{\prime}\right)$ of $G$ over $A$. Here $\mathfrak{p}^{\prime}$ is a non-zero prime ideal of $A^{\prime}$ so that if $p$ is the unique prime number in $\mathfrak{p}^{\prime}$ then $H$ is a $p$-hypoelementary subgroup of $G$ and $b$ is a generator of $H / O_{p}(H)$ where $O_{p}(H)$ is the largest normal $p$-subgroup of $H$.

Congruences. For any prime number q, we have

$$
\Phi_{L}\left(H, b, \mathfrak{p}^{\prime}\right) \equiv \Phi_{L}\left(O^{q}(H), b_{q^{\prime}}, \mathfrak{p}^{\prime}\right) \bmod \mathfrak{q}^{\prime}
$$

where $O^{q}(H)$ is the smallest normal subgroup of $H$ with $H / O^{q}(H)$ a q-group, $b_{q^{\prime}}$ is the $q^{\prime}$-part of the element $b$, and $\mathfrak{q}^{\prime}$ is any prime ideal above $q$.

Proof. Notations are consistent with those used in [3]. Let $i_{\mathfrak{p}^{\prime}}: A^{\prime} \rightarrow A_{\mathfrak{p}^{\prime}}^{\prime}$ be the inclusion of $A^{\prime}$ in its completion at $\mathfrak{p}^{\prime}$, and let $\mathfrak{p}=\mathfrak{p}^{\prime} \cap A$. Denote the $A_{\mathfrak{p}} G$-module $A_{\mathfrak{p}} \otimes_{A} L$ by $M$ for simplicity. Since $H$ is $p$-hypoelementary, $O_{p}(H)$ is the normal $p$ Sylow subgroup of $H$. Decompose the restriction $M_{H}$ of $M$ to $H$ as $M_{H} \simeq M^{\prime} \oplus M^{\prime \prime}$, where the vertices of the indecomposable $A_{\mathfrak{p}} H$-summands of $M^{\prime}$ are $O_{p}(H)$, and

Received by the editors September 7, 1995.

1991 Mathematics Subject Classification. Primary 20C10; Secondary 19A22, 20 C15.

(c)1997 American Mathematical Society 
the vertices of $M^{\prime \prime}$ are proper subgroups of $O_{p}(H)$. By the definition of $\Phi_{L}$ (cf. [3] (2.1)), we have

$$
i_{\mathfrak{p}^{\prime}} \Phi_{L}\left(H, b, \mathfrak{p}^{\prime}\right)=\text { trace of } b \text { acting on } M^{\prime}
$$

If the action of $b$ on $M^{\prime}$ has eigenvalues $\lambda_{1}, \ldots, \lambda_{r}$ in $A_{\mathfrak{p}^{\prime}}^{\prime}$, then $\Phi_{L}\left(H, b, \mathfrak{p}^{\prime}\right)=\sum_{i} \xi_{i}$, where $\xi_{i}$ is the preimage of $\lambda_{i}$ under $i_{\mathfrak{p}^{\prime}}$. We will call this the pretrace of $b$ on $M^{\prime}$ for convenience.

Denote $O_{p}\left(O^{q}(H)\right)=O_{p}(H) \cap O^{q}(H)$ by $Q$, and further decompose $M^{\prime \prime}$ as $M^{\prime \prime} \simeq M_{1}^{\prime \prime} \oplus M_{2}^{\prime \prime}$, where the vertices of the indecomposable $A_{\mathfrak{p}} H$-summands of $M_{1}^{\prime \prime}$ contain $Q$ and those of $M_{2}^{\prime \prime}$ do not.

Since every indecomposable $A_{\mathfrak{p}} H$-summand of $M^{\prime} \oplus M_{1}^{\prime \prime}$ has vertex $P$ between $Q$ and $O_{p}(H)$ from the above decomposition of $M_{H}$, it is an $A_{\mathfrak{p}} H$-summand of $\operatorname{ind}_{P}^{H}\left(A_{\mathfrak{p}}\right)$ by [3](1.1). Its restriction to $O^{q}(H)$ is then an $A_{\mathfrak{p}} O^{q}(H)$-summand of $\operatorname{ind}_{Q}^{O^{q}(H)}\left(A_{\mathfrak{p}}\right)$ by Mackey decompositon, hence has vertex $Q$. Every indecomposable summand of the restriction $\left(M_{2}^{\prime \prime}\right)_{O^{q}(H)}$ has vertex properly contained in $Q$ as the vertex can only drop after restriction. Therefore from the above decomposition of $M_{H}$, the restriction of $M$ to $O^{q}(H)$ has the decomposition $M_{O^{q}(H)} \simeq$ $\left(M^{\prime} \oplus M_{1}^{\prime \prime}\right)_{O^{q}(H)} \oplus\left(M_{2}^{\prime \prime}\right)_{O^{q}(H)}$, where the vertices of the indecomposable $A_{\mathfrak{p}} O^{q}(H)$ summands of $\left(M^{\prime} \oplus M_{1}^{\prime \prime}\right)_{O^{q}(H)}$ are $Q$, and the vertices of $\left(M_{2}^{\prime \prime}\right)_{O^{q}(H)}$ are proper subgroups of $Q$. Again by the definition of $\Phi_{L}$, applied to $\left(O^{q}(H), b_{q^{\prime}}, \mathfrak{p}^{\prime}\right)$, we obtain

$$
\Phi_{L}\left(O^{q}(H), b_{q^{\prime}}, \mathfrak{p}^{\prime}\right)=\text { pretrace of } b_{q^{\prime}} \text { acting on } M^{\prime} \oplus M_{1}^{\prime \prime} .
$$

Now the congruence follows from

Claim. i) pretrace of $b$ on $M^{\prime} \equiv$ pretrace of $b_{q^{\prime}}$ on $M^{\prime} \bmod \mathfrak{q}^{\prime}$;

ii) pretrace of $b_{q^{\prime}}$ on $M_{1}^{\prime \prime} \equiv 0 \bmod \mathfrak{q}^{\prime}$.

Proof of Claim. i) If $m$ is a sufficiently large power of $q$, we have $b^{m}=b_{q^{\prime}}^{m}$, and the eigenvalues of $b^{m}$ on $M^{\prime}$ have preimages $\xi_{1}^{m}, \ldots, \xi_{r}^{m}$ under $i_{\mathfrak{p}^{\prime}}$. Hence

$$
(\text { pretrace of } b)^{m}=\left(\sum_{i} \xi_{i}\right)^{m} \equiv \sum_{i} \xi_{i}^{m}=\text { pretrace of } b^{m} \bmod \mathfrak{q}^{\prime}
$$

and, for the same reason,

$$
\left(\text { pretrace of } b_{q^{\prime}}\right)^{m} \equiv \text { pretrace of } b_{q^{\prime}}^{m} \bmod \mathfrak{q}^{\prime} .
$$

Combining gives

$$
(\text { pretrace of } b)^{m} \equiv\left(\text { pretrace of } b_{q^{\prime}}\right)^{m} \bmod \mathfrak{q}^{\prime}
$$

from which i) follows.

ii) We may assume $M_{1}^{\prime \prime} \neq 0$. Then $Q \subsetneq O_{p}(H)$, hence $p$ must be equal to $q$, and $H / Q=\left(O_{p}(H) / Q\right) \times\left(O^{q}(H) / Q\right)$ is nilpotent.

Since $Q$ acts trivially on $M_{1}^{\prime \prime}$ by [3](1.1), $M_{1}^{\prime \prime}$ can be considered as an $A_{\mathfrak{p}} H / Q$ module. By $[4] \S 2$, this module has the structure,

$$
M_{1}^{\prime \prime} \simeq \sum_{j} N_{j} \otimes_{A_{\mathfrak{p}}} \operatorname{ind}_{D_{j}}^{O_{p}(H) / Q}\left(A_{\mathfrak{p}}\right)
$$

for some $A_{\mathfrak{p}} O^{q}(H) / Q$-lattices $N_{j}$ and some $p$-subgroups $D_{j}$ of $O_{p}(H) / Q$. These $D_{j}$ are actually the vertices of $M_{1}^{\prime \prime}$, hence are properly contained in $O_{p}(H) / Q$. 
If the eigenvalues of $b_{q^{\prime}}$ on $N_{j}$ have preimages $\xi_{1}^{(j)}, \ldots, \xi_{r_{j}}^{(j)}$ under $i_{\mathfrak{p}^{\prime}}$, then the eigenvalues of $b_{q^{\prime}}$ on $N_{j} \otimes_{A_{\mathfrak{p}}} \operatorname{ind}_{D_{j}}^{O_{p}(H) / Q}\left(A_{\mathfrak{p}}\right)$ have preimages $\xi_{1}^{(j)}, \ldots, \xi_{r_{j}}^{(j)}$ each repeated $\left|O_{p}(H) / Q: D_{j}\right|$ times. Thus

$$
\text { pretrace of } b_{q^{\prime}} \text { on } M_{1}^{\prime \prime}=\sum_{j}\left|O_{p}(H) / Q: D_{j}\right| \sum_{i} \xi_{i}^{(j)} \equiv 0 \quad \bmod p A^{\prime},
$$

as required. This completes the proof of the claim, hence of the congruence.

We want to examine the prime ideal spectrum $\operatorname{Spec}\left(\Omega_{A}(G)\right)$ of the commutative $\operatorname{ring} \Omega_{A}(G)$. Let $T_{G}(A)$ be the set of triples $\left(H, b, \mathfrak{p}^{\prime}\right)$, and $\left(A^{\prime}\right)^{T_{G}(A)}$ the ring of all maps on triples with values in $A^{\prime}$. Since the ring homomorphism $\Phi: \Omega_{A}(G) \rightarrow$ $\mho_{A}(G)$ has a nilpotent kernel [3], and $\mho_{A}(G)$ is a subring of $\left(A^{\prime}\right)^{T_{G}(A)}$ with finite Z-rank, it induces the surjection

$$
\operatorname{Spec}\left(\left(A^{\prime}\right)^{T_{G}(A)}\right) \stackrel{\text { going-down }}{\longrightarrow} \operatorname{Spec}(\operatorname{im} \Phi) \stackrel{\Phi^{-1}}{\longrightarrow} \operatorname{Spec}\left(\Omega_{A}(G)\right) .
$$

$\operatorname{Spec}\left(A^{\prime}\right)$ consists of the ideal 0 and the maximal ideals of $A^{\prime}$. The spectrum of $\left(A^{\prime}\right)^{T_{G}(A)}$ can be identified with $T_{G}(A) \times \operatorname{Spec}\left(A^{\prime}\right)$ : with each $T \in T_{G}(A)$ and each $\mathfrak{q}^{\prime} \in \operatorname{Spec}\left(A^{\prime}\right)$ we associate the prime ideal $\mathfrak{q}_{T}^{\prime}$ consisting of those $f \in\left(A^{\prime}\right)^{T_{G}(A)}$ such that $f(T) \in \mathfrak{q}^{\prime}$. The image of $\mathfrak{q}_{T}^{\prime}$ in $\operatorname{Spec}\left(\Omega_{A}(G)\right)$ is the prime ideal $P_{\mathfrak{q}^{\prime}, T}$ corresponding to the prime ideal $\mathfrak{q}_{T}^{\prime} \cap \operatorname{im} \Phi$ in $\operatorname{im} \Phi$ by $\Phi^{-1}$, i.e.

$$
P_{\mathfrak{q}^{\prime}, T}=\left\{x \in \Omega_{A}(G): \Phi_{x}(T) \in \mathfrak{q}^{\prime}\right\} .
$$

Lemma. With above notation, then

(1) $P_{0, T} \subset P_{\mathfrak{q}^{\prime}, T}$;

(2) If $\mathfrak{q}^{\prime}$ is a maximal ideal of $A^{\prime}$ above a prime number $q$ and $T=\left(H, b, \mathfrak{p}^{\prime}\right)$ is a triple, we denote the triple $\left(O^{q}(H), b_{q^{\prime}}, \mathfrak{p}^{\prime}\right)$ by $T^{q}$. Then $P_{\mathfrak{q}^{\prime}, T}=P_{\mathfrak{q}^{\prime}, T^{q}}$.

Proof. (1) is clear.

(2) By the congruences we have $\Phi_{x}(T) \equiv \Phi_{x}\left(T^{q}\right) \bmod \mathfrak{q}^{\prime}$ for $x \in \Omega_{A}(G)$. Thus $x \in P_{\mathfrak{q}^{\prime}, T} \Longleftrightarrow \Phi_{x}(T) \in \mathfrak{q}^{\prime} \Longleftrightarrow \Phi_{x}\left(T^{q}\right) \in \mathfrak{q}^{\prime} \Longleftrightarrow x \in P_{\mathfrak{q}^{\prime}, T^{q}}$

Corollary. $\operatorname{Spec}\left(\Omega_{A}(G)\right)$ is connected.

Proof. Let $C$ be the connected component of the point $P_{0,(1)}$ in $\operatorname{Spec}\left(\Omega_{A}(G)\right)$ where (1) is the cyclic triple (cf. [3] §3) of the trivial subgroup. By (1) of the Lemma, the closure $\overline{\left\{P_{0, T}\right\}}$ contains $P_{\mathfrak{q}^{\prime}, T}$ for all $\mathfrak{q}^{\prime}$. So it suffices to show that $C$ contains $\overline{\left\{P_{0, T}\right\}}$ for every triple $T$. We proceed by induction on the order of the subgroup $H$ appearing in the triple $T=\left(H, b, \mathfrak{p}^{\prime}\right)$.

If $H$ is trivial this follows by the definition of $C$ so we suppose $H$ is non-trivial. Choose a prime number $q$ so $O^{q}(H) \subsetneq H$, and a prime ideal $\mathfrak{q}^{\prime}$ of $A^{\prime}$ containing $q$. By (2) and (1) of the Lemma we have $P_{\mathfrak{q}^{\prime}, T}=P_{\mathfrak{q}^{\prime}, T^{q}}$, hence $\overline{\left\{P_{0, T}\right\}} \cap \overline{\left\{P_{0, T^{q}}\right\}}$ is not empty, and $\overline{\left\{P_{0, T}\right\}} \cup \overline{\left\{P_{0, T^{q}}\right\}}$ is connected. But $\overline{\left\{P_{0, T^{q}}\right\}} \subseteq C$ by the induction hypothesis, and therefore $\overline{\left\{P_{0, T}\right\}} \subseteq C$.

\section{ACKNOWLEDGEMENT}

I thank Alfred Weiss for his encouragement and interest in this work. 


\section{REFERENCES}

1. A. Dress, A characterization of solvable groups, Math. Z. 110 (1969), 213-217. MR 40:1491

2. J. P. Serre, Linear Representations of Finite Groups, Springer-Verlag, New York, 1977. MR 56:8675

3. X. Wang, A. Weiss, Permutation Summands over Z, J. Number Theory 47 (1994), 413-434. MR 95d:20008

4. A. Weiss, Torsion units in integral group rings, J. Reine Angew. Math. 415 (1991), 175-187. MR 92c:20009

Department of Mathematical Sciences, University of Alberta, Edmonton, Alberta, CANada T6G 2G1

Current address: Department of Computer Science, University of Toronto, Toronto, Ontario, Canada M5S 1A4

E-mail address: xywang@cs.toronto.edu 